
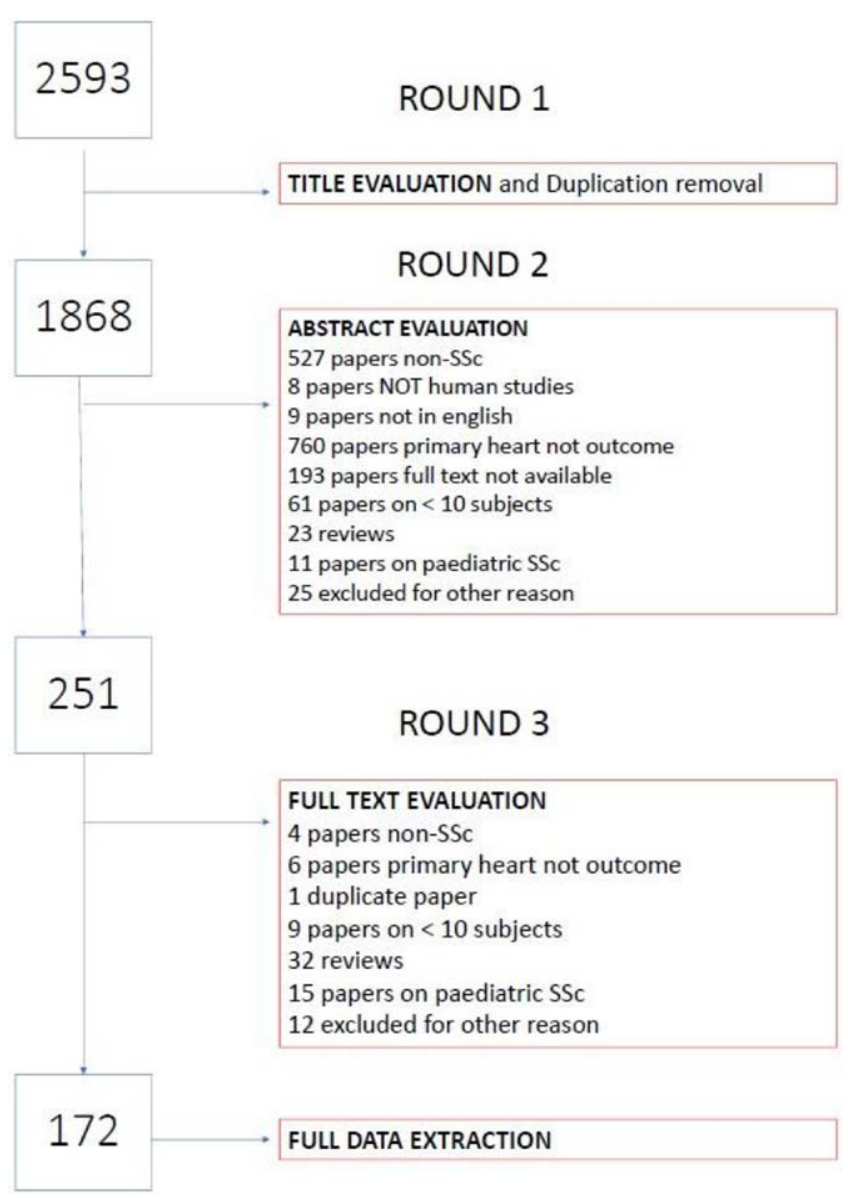

Conclusion: Using a SLR and modified nominal technique, we have developed a preliminary $\mathrm{pSScHI}$ consensus-based definition and started a validation process for it to be used in clinical research and clinical practice.

Acknowledgments: Aleksandra Djokovic, Giacomo De Luca, Raluca B. Dumitru, Alessandro Giollo, Marija Polovina, Yossra Atef Suliman, Kostantinos Bratis, Alexia Steelandt, Ivan Milinkovic, Anna Baritussio, Ghadeer Hasan, Anastasia Xintarakou, Yohei Isomura, George Markousis-Mavrogenis, Silvia Bellando-Randone, Lorenzo Tofani, Sophie Mavrogeni, Luna Gargani, Alida L.P. Caforio, Carsten Tschoepe, Arsen Ristic, Karin Klingel, Sven Plein, Elijah Behr, Yannick Allanore, Masataka Kuwana, Christopher Denton, Daniel E. Furst, Dinesh Khanna, Thomas Krieg, Renzo Marcolongo.

Disclosure of Interests: Cosimo Bruni Speakers bureau: Actelion, Eli Lilly, Maya H Buch Grant/research support from: Pfizer, Roche, and UCB, Consultant of: Pfizer; AbbVie; Eli Lilly; Gilead Sciences, Inc.; Merck-Serono; Sandoz; and Sanofi, Petar Seferovic: None declared, Marco Matucci-Cerinic Grant/research support from: Actelion, MSD, Bristol-Myers Squibb, Speakers bureau: Acetelion, Lilly, Boehringer Ingelheim

DOI: 10.1136/annrheumdis-2020-eular.1964

\section{AB0557 CO-EXISTENCE OF SYSTEMIC SCLEROSIS HALLMARK AUTOANTIBODIES ASSOCIATES WITH DISTINCT CLINICAL PHENOTYPE}

C. Campochiaro ${ }^{1,2}$, K. Clark' ${ }^{2}$, L. Host ${ }^{2}$, A. Sarı ${ }^{2}$, S. Nihtyanova ${ }^{2}$, C. Denton ${ }^{2}$, V. Ong ${ }^{2}{ }^{1}$ San Raffaele Scientific Institute - Vita-Salute San Raffaele University, Unit Immunology, Rheumatology, Allergy and Rare Diseases, Milan, Italy; ${ }^{2}$ Centre for Rheumatology and Connective Tissue Diseases, UCL Medical School, Royal Free Campus., London, United Kingdom

Background: Systemic sclerosis (SSc) is typically manifests with distinct SSc-specific antibodies (SSc-Abs): anti-topoisomerase I (ATA), anti-centromere (ACA), anti-RNA polymerase III (ARA), anti-U3RNP (U3RNP), anti-U1RNP (U1RNP), anti-PmScl (PmScl), anti-Ku (Ku) and anti-Th/TO (Th/T0), each being characterised by different clinical features and prognosis. The presence of $>1$ SSc-Abs is rare with minimum data about these patients' clinical phenotype. Objectives: To describe and compare the clinical features of SSc patients with $>1$ SSc-Ab
Methods: The autoantibody profiles of 2799 SSc patients from February 2001 to June 2017 were retrospectively reviewed. Patients with $>1$ SSc-Abs were identified. Clinical features were collected and compared to historical cohorts of SSc patients with single SSc-Ab positivity. Patients were excluded if treated prior to their immunology test with rituximab, iv immunoglobulins or stem cell transplantation. Statistical analysis was performed using Fisher exact test.

Results: 72 patients $(2.6 \%)$ with $>1$ SSc-Ab were identified. Full clinical data were available for 63 patients. 60 patients $(2.1 \%)$ had double Ab positivity and 3 patients had triple Ab positivity $(0.1 \%)$. $13 \mathrm{Ab}$ combinations were present. U1RNP and ATA was the most frequent combination (35\%), patients were significantly younger ( 51.38 years) than both U1RNP ( 58.64 years, $p=0.050)$ and ATA (62.03 years, $p=0.002$ ) patients and more commonly of diffuse subset (dcSSc) ( $p=0.001$ and $p=0.041$ respectively). Compared to ATA patients overlap features were more frequent ( $43 \%$ vs $15 \%, p=0.004$ ) including inflammatory arthritis $(p=0.025)$ and myositis $(p=0.013)$ (Table 1). U1RNP and ACA had a significantly higher prevalence of pulmonary arterial hypertension compared to U1RNP ( $p=0.039)$ and ACA $(p=0.022)$ patients, and compared to ACA patients they were younger ( $57.88 \mathrm{vs} 68.75, p=0.015$ ) with a higher incidence of myositis $(p=0.001)$. U1RNP and ARA patients were more frequently dcSSc subtype compared to U1RNP patients (75\% vs $21 \%, p=0.040$ ). U1RNP and PmScl patients had a higher prevalence of myositis compared to U1RNP patients $(p=0.006)$. ATA and ACA patients behaved similarly to ATA patients with a significantly higher prevalence of lung fibrosis $(p=0.006)$ and myositis $(p=0.041)$ compared to ACA. ACA and PmScl $(7 \%)$ had higher prevalence of myositis compared to ACA patients $(p=0.04)$.

Table 1. Frequency of clinical features in some of the double antibody group combinations, compared to our cohort of patients with only one of the SSc specific antibody. Significant $p$ values $(<0.05)$ highlighted in bold. ILD (interstitial lung disease), PAH (pulmonary arterial hypertension), SRC (scleroderma renal crisis).

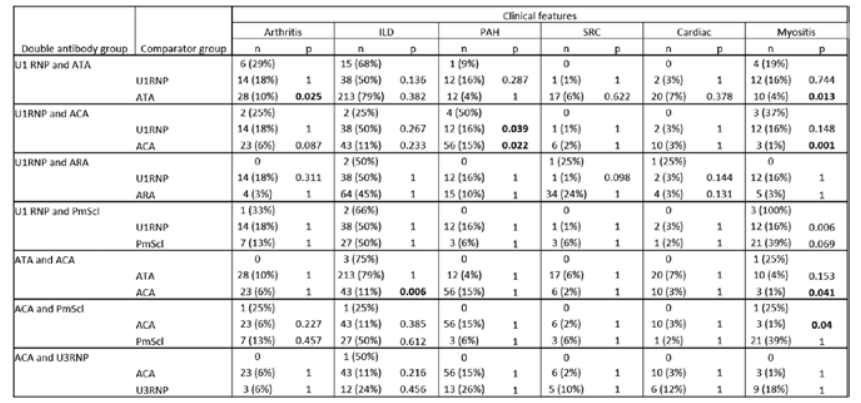

Conclusion: Coexistence of hallmark autoantibodies is exceedingly rare in SSc patients. When combined, both SSc-Abs have the potential to synergistically interact and modify the clinical phenotype.

Disclosure of Interests: Corrado Campochiaro Speakers bureau: Novartis, Pfizer, Roche, GSK, SOBI, Kristina Clark: None declared, Lauren Host: None declared, Alper Sarl: None declared, Svetlana Nihtyanova: None declared, Christopher Denton Grant/research support from: GlaxoSmithKline, CSL Behring, and Inventiva, Consultant of: Medscape, Roche-Genentech, Actelion, GlaxoSmithKline, Sanofi Aventis, Inventiva, CSL Behring, Boehringer Ingelheim, Corbus Pharmaceuticals, Acceleron, Curzion and Bayer, Voon Ong: None declared DOI: 10.1136/annrheumdis-2020-eular.3500

\section{AB0558 RISK FACTORS FOR THE DEVELOPMENT OF BREAST CANCER IN SYSTEMIC SCLEROSIS}

C. Carbonell ${ }^{1}$, A. J. Chamorro ${ }^{1}$, M. Marcos ${ }^{1}$, A. Guillén del Castillo², D. Colunga Argüelles $^{3}$, M. Rubio-Rivas ${ }^{4}$, A. Argibay ${ }^{5}$, B. Marí-Alfonso ${ }^{6}$, A. Marín Ballvé ${ }^{7}$, A. Castro ${ }^{8}$, A. B. Madroñero-Vuelta ${ }^{9}$, E. L. Callejas-Moraga ${ }^{6}$, V. Fonollosa-Pla ${ }^{2}$, C. P. Simeón-Aznar ${ }^{2}$, O. B. O. R. I. Autoimmune Diseases Study Group (Geas) ${ }^{10}$ ${ }^{1}$ Hospital Clínico Universitario de Salamanca-USAL-IBSAL, Salamanca, Spain; ${ }^{2}$ Hospital Universitario Vall d'Hebron, Unit of Autoimmune Diseases, Internal Medicine, Barcelona, Spain; ${ }^{3}$ Hospital Universitario Central de Asturias, Internal Medicine, Asturias, Spain; ${ }^{4}$ Hospital Universitario de Bellvitge-IDIBELL, Unit of Autoimmune Diseases, Internal Medicine, Barcelona, Spain; ${ }^{5}$ Complejo Hospitalario Universitario de Vigo, Unit of Autoimmune Diseases, Internal Medicine, Pontevedra, Spain; ${ }^{6}$ Corporación Sanitaria Universitaria Parc Taulí, Internal Medicine, Barcelona, Spain; ${ }^{7}$ Hospital Clínico Universitario Lozano Blesa, IIS Aragón, Unit of Autoimmune Diseases, Internal Medicine, Zaragoza, Spain; ${ }^{8}$ Hospital Universitario Sant Joan, Internal Medicine, Tarragona, Spain; 
${ }^{9}$ Hospital General San Jorge, Internal Medicine, Huesca, Spain; ${ }^{10}$ Sociedad Española de Medicina Interna, Madrid, Spain

Background: Prior literature shows a global increase of cancer risk among patients with systemic sclerosis (SSc). Although breast cancer (BC) is one of the most frequent malignancies in SSc patients, the characteristics of this neoplasm among SSc patients is not well established and it is uncertain whether SSc significantly increases the risk of this cancer.

Objectives: Describe the characteristics and risk factors for BC among Spanish patients with SSc.

Methods: Ambispective and multicenter study of patients with a diagnosis of SSc according to ACR/EULAR criteria and/or LeRoy classification included in the Spanish Scleroderma Registry (RESCLE) from 2006 to 2018. Characteristics of patients with $\mathrm{BC}$ were described and incidence was compared with that of the general population.

Results: Among 1930 patients with SSc, 206 (10.7\%) had cancer. BC was the most frequent tumor location (47 patients of 206 with cancer [22.8\%]), followed by lung cancer $(29,14.1 \%)$. The risk of BC was increased in patients with SSc compared to the general population (standardized incidence ratio [SIR] 1.31; $95 \% \mathrm{Cl} 1.10-1.54 ; P=0.003)$.

The comparison of patients with $\mathrm{BC}$ and those without cancer showed that patients with BC had older age at diagnosis of SSc (50.9 vs 45.9 years, respectively; $P=0.004$ ), were more frequently diagnosed of interstitial lung disease (ILD) $(30 / 47$ [63.8\%] vs $694 / 1714$ [40.5]; $P=0.002)$ and pulmonary hypertension (12/24 [50.0\%] vs $262 / 917$ [28.6\%]; $P=0.037$ ), had higher frequency of puffy hands as the first manifestation of SSc (4/45 [8.9\%] vs 38/1664 [2.3 $\%$ ]; $P=0.023)$ and had more frequently primary biliary colangitis (PBC) $(7$ /46 [15.2\%] vs $72 / 1708$ [4.2\%]; $P=0.004)$. Regarding autoimmunity profile, patients with SSc and BC had a significantly higher presence of anti-Ro (11/44 [25.0\%] vs 214/1528 [14.0\%]; $P=0.049)$ and anti-mitochondrial antibodies (7/28 [25.0\%] vs $96 / 837$ [11.5\%]; $P=0.039$ ). Multivariable regression analysis showed an independent association between the puffy hands (OR $=6.40 ; 95 \%$ Cl 1.73-23.60; $P=0.005)$, diagnosis of $\mathrm{PBC}(\mathrm{OR}=5.70 ; 95 \% \mathrm{Cl} 2.16-15.07$; $P=0.001)$, presence of ILD $(\mathrm{OR}=3.29,95 \% \mathrm{Cl} 1.69-6.39 ; P<0.001)$ and the presence of the anti-Ro antibody (OR $2.14 ; 95 \% \mathrm{Cl} 1.01-4.56 ; P=0.048$ ) with the presence of $\mathrm{BC}$.

Conclusion: BC risk was increased in patients with SSc. The development of ILD, PBC, the presence of anti Ro and puffy hands as the first clinical manifestation of SSc were identified as independent factors associated with the development of $B C$ in our cohort.

References: Colaci $\mathrm{M}$ et al. Breast cancer in systemic sclerosis: results of a cross-linkage of an Italian Rheumatologic Center and a population-based Cancer Registry and review of the literaure. Autoimmun Rev. 2014;13(2):132-7.

Zhang JQ et al. The risk of cancer development in systemic sclerosis: a meta-analysis. Cancer Epidemiol. 2013;37(5):523-7.

Disclosure of Interests: Cristina Carbonell: None declared, Antonio-J Chamorro: None declared, Miguel Marcos: None declared, Alfredo Guillén del Castillo: None declared, Dolores Colunga Argüelles Consultant of: Actelion pharmaceuticals, GSK, MSD., Manuel Rubio-Rivas: None declared, Ana Argibay: None declared, Begoña Marí-Alfonso: None declared, Adela Marín Ballvé: None declared, Antoni Castro Consultant of: Actelion pharmaceuticals, GSK, MSD., Ana Belén Madroñero-Vuelta: None declared, Eduardo L. Callejas-Moraga: None declared, Vicent Fonollosa-Pla Consultant of: Actelion pharmaceuticals, GSK, MSD., Carmen Pilar Simeón-Aznar Consultant of: Actelion pharmaceuticals, GSK, MSD., on behalf of RESCLE Investigators, Autoimmune Diseases Study Group (GEAS): None declared

DOI: 10.1136/annrheumdis-2020-eular.2016

\section{\begin{tabular}{l|l} 
AB0559 & ARRHYTHMIAS AND LEFT VENTRICULAR
\end{tabular} DYSFUNCTION IN PATIENTS WITH SYSTEMIC} SCLEROSIS.

L. López-Núñez ${ }^{1}$, I. Carrión Barberà ${ }^{1}$, I. Padró ${ }^{1}$, L. Molina ${ }^{2}$, A. Pros ${ }^{1} .{ }^{1}$ Hospital del Mar, Rheumatology, Barcelona, Spain; ${ }^{2}$ Hospital del Mar, Cardiology, Barcelona, Spain

Background: Cardiac involvement in systemic sclerosis (SS) is common and one of the leading causes of mortality (up to $31 \%$ ). Usually, it appears early and in a silent way. It can affect any cardiac structure and present with various manifestations. Cardiac disease worsens the prognosis and increases mortality, so it should be kept in mind in these patients.

Objectives: Study the prevalence of arrhythmias in patients with SS, not affected with pulmonary arterial hypertension (PAH) or other cardiac diseases, and establish its association with left ventricular (LV) dysfunction and heart dysautonomia (HD), as well as with other variables that could predict the development of this complication.
Methods: Prospective study of a cohort of patients with SS, excluding those with heart disease, $\mathrm{PAH}$ or cardiovascular risk factors. All underwent a clinical assessment, blood test with cardiac biomarkers, electrocardiogram (ECG), Holter 24h (HLT) and echocardiogram (TTE), interpreted by an expert cardiologist blind about the patients. Arrhythmias were classified as clinically significant arrhythmias (CSA) or clinically nonsignificant arrhythmias (CNSA) by ECG and HLT. LV diastolic dysfunction (LVDD) was defined as E/e'> 8, LV systolic dysfunction (LVSD) as a global longitudinal strain $<20 \%$ and HD as a SDNN $<100 \mathrm{~ms}$. Demographic, clinical and biological data were collected. A follow-up was performed at $6.2 \pm 0.9$ years. Statistical analysis was performed using SPSS 23 IBM@.

Results: 36 patients were included: age $56.7 \pm 12.3$ years $(y)$, male / female $35 / 1$, disease duration $7 \pm 4.1$ y. $66 \%$ belonged to the limited SS subtype, $66.6 \%$ were anti-centromere+, 25\% anti-topoisomerase, 2.7\% anti-PM/Scl and $2.7 \%$ anti-RNA polymerase III+. Raynaud was present in a $100 \%$, telangiectasia in $55.6 \%$ and interstitial lung disease in $36.1 \%$. The modified Rodnan skin score (mRSS) was $\leq$ moderate (0-29 points) in $55.6 \%$; $27.8 \%$ had presented digital ulcers that required prostaglandins.

27.8\% had LVDD, 22\% LVSD, 11.1\% LVDD + SD and 16.7\% HD. 50\% (18/36) of patients had ECG alterations, of which $44 \%$ corresponded to CSA (Table 1) and, $55.6 \%(20 / 36)$ HLT alterations, of which $75 \%$ were CSA (Table 2). 3/36 patients had both HLT and ECG CSA. In 1 patient, impaired LV ejection fraction was detected; in none, valvular disease. $38.8 \%$ had elevated NT-proBNP and $13.9 \%$ troponin $\mathrm{T}(\mathrm{TnT})$. No correlation was found between any parameters and CSA.

A correlation was found between mRSS and DLCO $(p=0.002), D L C O$ and digital ulcers $(p=0.001)$, and mRSS and digital ulcers $(p=0.005)$. A correlation was also found between elevated NT-proBNP and TnT $(p=0.006)$ and between elevated NT-proBNP and LVDD $(\mathrm{p}=0.049)$.

At follow-up after $6.2 \pm 0.9$ y, 2 patients had died: 1 of neoplasia and 1 of severe biventricular dysfunction 5.2 y after the study.

Conclusion: Our data confirm a high prevalence of ventricular arrhythmias and left dysfunction in patients with SS, without heart disease, cardiorespiratory symptoms or HAP, being up to $75 \%$ of the arrhythmias CSA. The lack of correlation between CSA and LVSD or DD indicates that arrhythmias could be due, not only to a supposed structural alteration of the myocardium, but to a primary and early cardiac involvement in SS. In addition, the lack of correlation between CSA in ECG and HLT reinforces the importance of a complete cardiac evaluation in these patients to rule out silent cardiac involvement.

Table 1. ECG abnormalities. *CSA.

\begin{tabular}{lcc}
\hline Alterations & \# Patients \\
\hline Major & Complete Left Bundle Branch Block (BBB)* & 1 \\
Minor & Abnormal QRS prolongation in precordial leads & 1 \\
& Nonspecific ST-T wave changes & 3 \\
& Incomplete Left BBB & 3 \\
Other & Incomplete Right BBB & 4 \\
& QT prolongation & 11 \\
\hline
\end{tabular}

Table 2. HLT alterations. *CSA.

\begin{tabular}{lcc}
\hline Alterations & Subtype & $\begin{array}{c}\# \\
\text { Patients }\end{array}$ \\
\hline Supraventricular Extrasystoles & Uncommon & 7 \\
& Frequent $^{\star}$ & 3 \\
Nonsustained Supraventricular Tachycardia* & Mono/Polymorphic & 0 \\
Ventricular Extrasystoles & Benign & 7 \\
& No Benign & 4 \\
& Doublets & 4 \\
& Two Morphologies & 2 \\
1st-degree Atrioventricular Block & Accelerated Idioventricular & 1 \\
Sinus Tachycardia & Rhythm & 1 \\
& & 2 \\
\hline
\end{tabular}

Disclosure of Interests: LILIAN LÓPEZ-NÚÑEZ: None declared, Irene Carrión Barberà Grant/research support from: I received a grant from the Spanish Rheumatology Foundation (FER) and laboratories KERN PHARMA for a brief stay abroad., Isabel Padró: None declared, Lluis Molina: None declared, Ana Pros: None declared

DOI: 10.1136/annrheumdis-2020-eular.2874 